\title{
Polling Deregistration for Unlicensed PCS
}

\author{
Anthony R. Noerpel, Senior Member, IEEE, Li Fung Chang, Senior Member, IEEE, and Yi-Bing Lin
}

\begin{abstract}
This paper describes a polling deregistration protocol for a wireless access communications network which would support interoperability between licensed personal communications services (PCS) and unlicensed-PCS (UPCS). We show how the protocol can be efficiently implemented in the personal access communications system (PACS) for licensed PCS and in the PACS-Unlicensed Version B (PACS-UB). An analytical model is proposed to analyze the performance of the polling protocol. A cost function is derived, which can be used to estimate the optimal polling frequency.
\end{abstract}

\section{INTRODUCTION}

$\mathbf{T}$ WHE FCC has allocated the spectrum between 1850-1910 and 1930-1990 MHz for licensed personal communications services (PCS) and the band between 1920-1930 MHz for unlicensed PCS (UPCS). The use of the latter is for isochronous communications and a set of rules (an etiquette) has been published by the FCC to allow for different systems to share this spectrum. The isochronous operation is intended primarily for circuit-oriented voice and data applications. PACS-UB [2], [12], [13] is a version of the Joint Technical Committee's (JTC) PACS Air Interface Specification modified to conform to the FCC [1], [3] etiquette rules for the unlicensed spectrum.

An important and desirable feature of the new PCS technologies is the potential interoperability between both private and public wireless access using dual mode handsets which can operate in both the licensed PCS and UPCS spectrum [8], [15], [17]. A fast polling algorithm is described in this paper for both PACS and PACS-UB to facilitate interoperability between public and local (private) wireless access systems by enabling a wireless access network to keep track of a handset location as it moves between PCS venues deploying either licensed or unlicensed spectrum options. This would allow the network to successfully deliver a call. By periodically polling a handset, the local wireless system can know when a handset has left its coverage area or the system venue. In the case of a multitier implementation the wireless system can relay this information to an access manager [3] or a home location register (HLR) of a public PCS system as required.

Manuscript revised December 1995.

A. R. Noerpel is with Hughes Network Systems, Germantown, MD 20876 USA.

L. F. Chang is with Bellcore, Red Bank, NJ 07701 USA

Y.-B. Lin is with the Department of Computer Science and Information Engineering, National Chiao Tung University, Hsinchu, Taiwan, ROC (e-mail: liny@csie.nctu.edu.tw).

Publisher Item Identifier S 0733-8716(96)01951-8.
In network mobility management protocols such as IS-41 [5] and GSM [10] deregistration can be efficiently achieved as part of the registration process. When a handset moves to a new registration area (RA), the visitor location register (VLR) of the RA sends a registration message to the HLR, and the old location is automatically deregistered at the HLR. (A deregistration is sent to the old VLR to cancel the obsolete VLR record.)

Polling deregistration is included in the PACS-UB air interface protocol to accommodate the situation when the unlicensed wireless local communication network is integrated with the licensed PCS systems such as intermediate tier and high tier microcellular and cellular phone networks. In such an environment, a multimode handset alternately switches its mode of operation as it moves between coverage areas. Ideally, the handset would always select to monitor the most economic service access option. To avoid a large number of registrations, when a handset is at the edge of coverage or in an area covered by high tier, intermediate tier and low tier (i.e., wireless local network), because of rapidly changing propagation conditions, it is practical for the network and handset to maintain registration areas for the three tiers independently. Thus a handset would only register to a tier when it entered the coverage area of a base station in a new registration area and not when it reverted to a different tier.

As the low or intermediate licensed tier radio system, PACS has two unique capabilities not shared by any other radio system specification. The PACS air interface protocol provides for handset alerting before call delivery routing. PACS also has more alerting channel capacity than any other radio system standard by an order of magnitude with virtually no alert blocking and low alerting delays even during busy hours. These capabilities enable the PACS system to interoperate with other types of wireless access systems in a cost effective manner.

Alerting the handset before routing the call is more economic than alerting after routing and allows the easy implementation of alternative call treatments such as voice mail, messages or even routing the call to another wireless or wireline access system. The handset can be multiply registered simultaneously to a high mobility, high tier, public radio system, an intermediate tier, public radio system deploying PACS, and a low-tier UPCS private radio system such as a wireless PBX, wireless Centrex or residential cordless system, simultaneously. An incoming call to the PCS subscriber can cause alerting on the PACS intermediate tier first. If the handset does not respond the call must be routed to the high 
tier or the low tier private system. However, it cannot be routed to both because of the limitations of these networks and air interfaces. Thus a method of deregistering from the low tier private system is desirable, since this would likely be the first choice alternative to the intermediate tier for call delivery. Polling deregistration is a practical and reliable method of determining if the handset is still present in the private system.

Implicit deregistration is a novel technique which can be used to save signaling messages on the network [9]. Whenever a handset moves from one RA to another within the same tier of service, it must register to the new VLR. Then new VLR signals the arrival of the handset to the HLR, which updates the user location record. The HLR then deregisters the user from the old VLR. When a call arrives for the user, the HLR is queried and the call is routed to the new registration area. The deregistration process to the old VLR is unnecessary from a call delivery point of view. It is only necessary to clean up old registration records and free up registration resources at the old VLR. The network deregistration signaling can be eliminated, i.e., the handset can be implicitly deregistered at the HLR from the old VLR, provided that old VLR has a method of cleaning up old registration records. Polling handsets is a method of determining whether the handset is still present within the registration area.

Polling is also specified in GSM [6] for database failure restoration. In PACS and PACS-UB, polling is used for both deregistration and failure restoration.

This paper describes the polling protocol and analyzes its limitations and effectiveness. A simple contention algorithm is used to avoid contention of poll responses. The detailed analysis of the contention algorithm can be found in [11]. In this paper, we focus on the polling protocol, the traffic analysis of this protocol, and provide guidelines to determine the polling frequency.

\section{The Polling Protocol}

While registered to an unlicensed system, a handset can be periodically polled by receiving an alert in the normal fashion, i.e., as if the network has an incoming call to be delivered to the handset. This alert is preceded by a prefix directive which indicates that the following alert value is being polled. Since there might be many radio port control units (RPCU) subtending a VLR or access manager in a large private system, a particular RPCU is not necessarily aware that a handset is being polled as the poll request originates from the VLR. The handset therefore responds to the alert by transmitting to the base station a message which contains as an information element the alert value and alert phase of the handset. Thus the RPCU knows that the handset is responding to a poll request and not to a call delivery alert. The RPCU responds with an acknowledgment message with the cause element set to "poll response only." In PACS, polling can take place in a few tens of milliseconds at the data link layer.

If the access manager or VLR in a large system, or the base station itself in a small system such as a residential cordless base station or wireless keyset, does not receive the polling response from the handset, it may try to poll the handset again, the number of polling retries being a system parameter. If there is no response from the handset within a timeout period, the system can deregister the handset. The HLR can be informed of this deregistration.

\section{Why POLLING?}

In Section I, we have shown that polling with implicit deregistation can be used to reclaim obsolete VLR records without creating extra signaling traffic between the HLR and the VLR. A more important role of polling was briefly described in Section I, and is elaborated in this section.

Consider a multitier system. When a handset enters a new RA in any tier, it registers and the network deregisters the handset from the old VLR of the same tier. If the new tier is a low tier UPCS system, then new call deliveries will be preferentially routed to the new RA in the new UPCS system. If the new RA is in the intermediate licensed tier using the PACS air interface protocol, then the network routes incoming calls to the intermediate tier. If the new RA is in the high tier system, then the network can optionally alert the HS on the intermediate tier by first holding the call in the advanced intelligent network (AIN) tandem switch [4] which triggers on the called party's mobile phone number. It should take this alert process from five to ten seconds to determine that the HS is not available in the intermediate tier and the call can then be routed to the high tier RA or a voice mail service as specified in the user profile. If a handset begins to monitor a new tier but is in the same RA for that tier then a new registration is not necessary. This scenario does not pose a problem for movement between the intermediate and high tiers because of the alerting capabilities of PACS. However, for movement between the low tier and either of the other two tiers, the handset will not automatically register because it detects the $\mathrm{RA}$ in that tier to which it is already registered. Therefore polling deregistration from the UPCS tier is desirable.

\section{Analysis of the Polling Protocol}

Figure 1 illustrates the timing relationship among the HLR, the base station (BS), and the handset (HS). We assume that the message transmission delays are zeros but this restriction can be easily relaxed in our model.

While in the UPCS system, the HS will be polled every $T$ seconds to confirm the location of the HS within the tier. Suppose that the user resides in the RA for a period of $t_{m}$. The BS polls the HS at time $t_{m}+t$ and does not receive any response (because the HS has left the RA). The BS waits for a timeout period $\Delta$, then deregisters the HS by sending a message to HLR. The period $X=t+\Delta$ is the time interval between when the HS leaves the RA and when HLR is informed the move of the HS. If $T$ is large, then it is likely that a call arrives during the period $X$, and the call will be delivered to the wrong RA. On the other hand, if $T$ is small, then the HS needs to respond often and each time the HS power is consumed. In a large venue such as a wireless PBX or Centrex system, there may also be a lower limit on $T$ because of the bandwidth limitations of the alerting channel. 


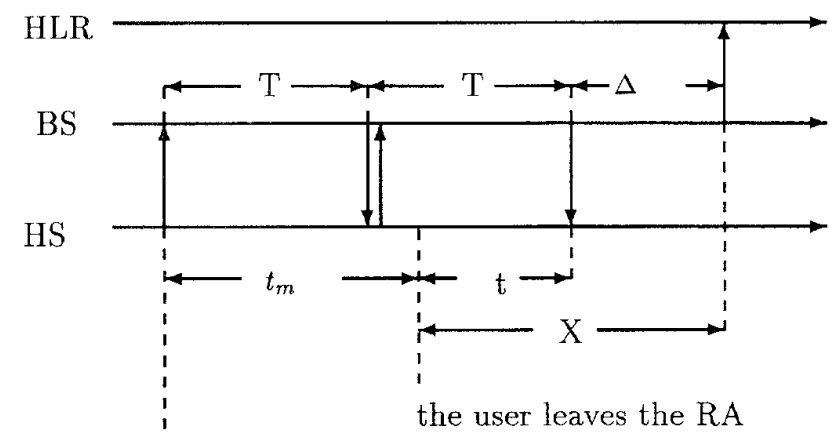

the user enters the RA

Fig. 1. The timing diagram.

Let $\alpha$ be the probability that a call arrives during $X$, and $\beta$ be the expected number of re-registration (polling response) messages sent from the HS per unit time. Then when a PCS system is designed, the value $T$ should be selected such that the cost

$$
C=\alpha+c \beta
$$

is minimized. For a particular UPCS network, economic analysis is required to estimate the weighted factor $c$. The economic analysis is beyond the scope of this paper. Instead, we will select some values of $c$ for the demonstration purpose. The probability $\alpha$ is expressed as (11) in Appendix A (in the equation, $\alpha^{*}=1-\alpha$ ) by assuming that $t_{m}$ has a general density function $f_{m}$.

If the user residual time in an RA has a Gamma distribution, then the probability is expressed as (15) in Appendix A. The Gamma density function $f_{m}\left(t_{m}\right)=f_{\gamma, \eta}\left(t_{m}\right)$ with the shape parameter $\gamma$ and the scale parameter $\eta[7]$ is expressed as

$$
f_{m}\left(t_{m}\right)=f_{\gamma, \eta}\left(t_{m}\right)=\frac{\eta^{\gamma} t_{m}^{\gamma-1} e^{-\eta t_{m}}}{\Gamma(\gamma)}
$$

where

$$
\Gamma(\gamma)=\int_{\tau=0}^{\infty} e^{-\tau} \tau^{\gamma-1} d \tau, \text { and } \gamma>0
$$

Depending upon the values of the parameters, it can be shaped to represent many distributions as well as measured data. For example, one may measure the user RA residual times in a real PCS network, and the measured data can be approximated by a Gamma distribution as the input to our polling model. Let $\mu$ be the mobility of a user (i.e., the rate that the user leaves an RA). In other words, $1 / \mu$ is the expected residual time of the user in an RA. For the Gamma residual time distribution, $\mu=\eta / \gamma$ and variance $V=1 / \gamma \mu^{2}$ [7]. For a fixed $\mu$, let $\alpha(V=v)$ be the value of $\alpha$ when the variance is $v$. For $\gamma=1,2$ and $3,(15)$ is rewritten as

$$
\begin{aligned}
& \alpha\left(V=1 / \mu^{2}\right)=1-\frac{\mu e^{-\lambda \Delta}\left(e^{-\lambda T}-e^{-\mu T}\right)}{(\mu-\lambda)\left(1-e^{-\mu T}\right)} \\
& \alpha\left(V=1 /\left(2 \mu^{2}\right)\right) \\
& \quad=1-\frac{4 \mu^{2} e^{-\lambda \Delta}}{(2 \mu-\lambda)^{2}\left(1-e^{-2 \mu T}\right)}
\end{aligned}
$$

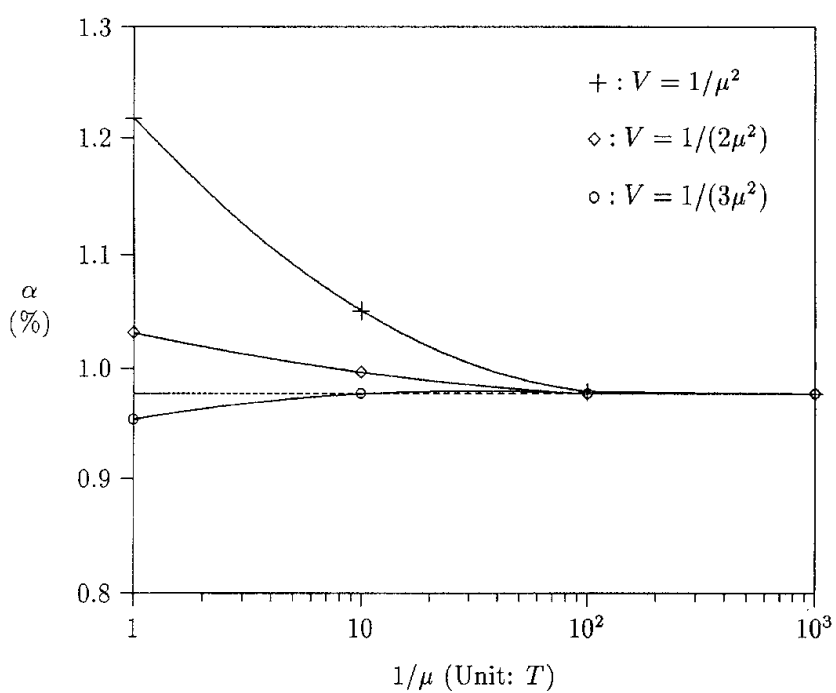

Fig. 2. Effect of $\mu$ on $\alpha[T=30 \mathrm{~s}, \lambda=1 /(2000 \mathrm{~s})]$.

$$
\begin{aligned}
& \times\left[\frac{e^{-2 \mu t}\left(e^{-\lambda T}-1\right)(2 \mu-\lambda) T}{\left(1-e^{-2 \mu T}\right)}+e^{-\lambda T}-e^{-2 \mu T}\right] \\
\alpha(V= & \left.1 /\left(3 \mu^{2}\right)\right) \\
= & 1-\frac{27 \mu^{3} e^{-\lambda \Delta}}{(3 \mu-\lambda)^{3}\left(1-e^{-3 \mu T}\right)} \\
& \times\left\{e^{-\lambda T}-e^{-3 \mu T}+\left(\frac{e^{-3 \mu T}}{1-e^{-3 \mu T}}\right)\right. \\
& \left.\times\left[e^{-\lambda T}-1-\frac{(3 \mu-\lambda) T}{2}+\frac{2(3 \mu-\lambda) T}{1-e^{-3 \mu T}}\right]\right\} .
\end{aligned}
$$

Fig. 2 plots $\alpha$ values based on (3). The figure indicates that for a large $1 / \mu$ value (e.g., $1 / \mu>100 T$ ), $\alpha$ is independent of the variance of the Gamma distribution. In Appendix B, we show that

$$
\lim _{\mu \rightarrow 0} \alpha=1-\frac{e^{-\lambda \Delta}\left(1-e^{-\lambda T}\right)}{\lambda T}
$$

which is independent of the $\gamma$ values (cf. the dashed line in Fig. 2). In fact, as $\mu \rightarrow 0$, the interval $t$ has a uniform distribution. This statement is proved as follows. If $t$ has a uniform distribution, then its density function is

$$
f(t)=\frac{1}{T} \quad \text { for } \quad 0 \leq t \leq T .
$$

For call arrival rate $\lambda$, the probability that no call arrives during $X$ is (see (9) in Appendix A) $e^{-\lambda X}$, and

$$
\alpha=1-\int_{t=0}^{T} \frac{1}{T} e^{-\lambda(t+\Delta)} d t=1-\frac{e^{-\lambda \Delta}\left(1-e^{-\lambda T}\right)}{\lambda T} .
$$

Equation (5) is the same as (4). Fig. 3 plots the $\alpha$ curves assuming $\mu \rightarrow 0$.

The value $\beta$ or the expected number of re-registration messages sent from the HS per unit time is expressed as (22) in Appendix $\mathrm{C}$ assuming that $t_{m}$ has a general distribution. 


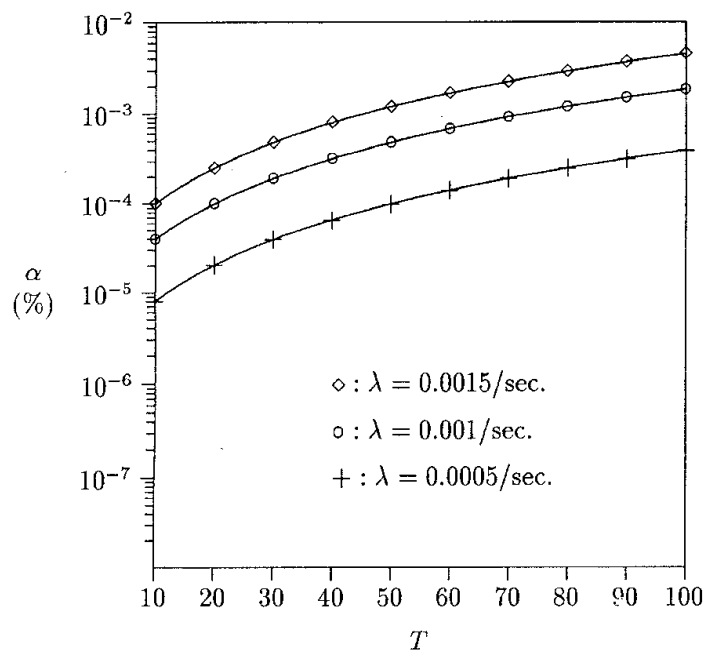

Fig. 3. Effect of $T$ on $\alpha(\mu \rightarrow 0)$.

If $t_{m}$ is Gamma distributed with nonnegative integer $\gamma$ value, then $\beta$ is expressed as (23) in Appendix C. For a fixed $\mu$, let $\beta(V=v)$ be the value of $\beta$ when the variance is $v$. For $\gamma=1,2$ and $3,(23)$ is rewritten as

$$
\begin{aligned}
& \beta\left(V=1 / \mu^{2}\right)=\frac{\mu e^{-\mu T}}{1-e^{-\mu T}} \\
& \beta\left(V=1 /\left(2 \mu^{2}\right)\right)=\frac{\mu e^{-2 \mu T}\left(1-e^{-2 \mu T}+2 \mu T\right)}{\left(1-e^{-2 \mu T}\right)^{2}} \\
& \beta\left(V=1 /\left(3 \mu^{2}\right)\right) \\
& \quad=\frac{\mu e^{-3 \mu T}}{1-e^{-3 \mu T}}\left[1+\frac{3 \mu T}{1-e^{-3 \mu T}}+\frac{(3 \mu T)^{3}\left(1+e^{-3 \mu T}\right)}{2\left(1-e^{-3 \mu T}\right)^{2}}\right] .
\end{aligned}
$$

Figure 4 plots $\beta$ values based on (6). The figure indicates that for a large $1 / \mu$ value (e.g., $1 / \mu>100 T$ ), $\beta$ is independent of the variance of the Gamma distribution. In Appendix B, we show that

$$
\lim _{\mu \rightarrow 0} \beta=\frac{1}{T} .
$$

Thus, for $1 / \mu=E\left[t_{m}\right]>100 T$, the cost function (1) is rewritten as

$$
C=1-\frac{e^{-\lambda \Delta}\left(1-e^{-\lambda T}\right)}{\lambda T}+\frac{c}{T}
$$

Figure 5 plots $C$ as a function of $T$ and $c$. In this figure, the bullets in the curves represent the cost for the optimal $T$ values. For $c=0.001$, the polling costs are very low and $T$ should be small, perhaps a few seconds for a residential cordless system and about $30 \mathrm{~s}$ for a large wireless Centrex or PBX system. For $c=10$, the polling costs are high and $T$ should be greater than $100 \mathrm{~s}$.

\section{CONCLUSION}

This paper describes a polling deregistration protocol for PCS or UPCS applications. We describe how the protocol can be efficiently implemented using the PACS-UB air interface

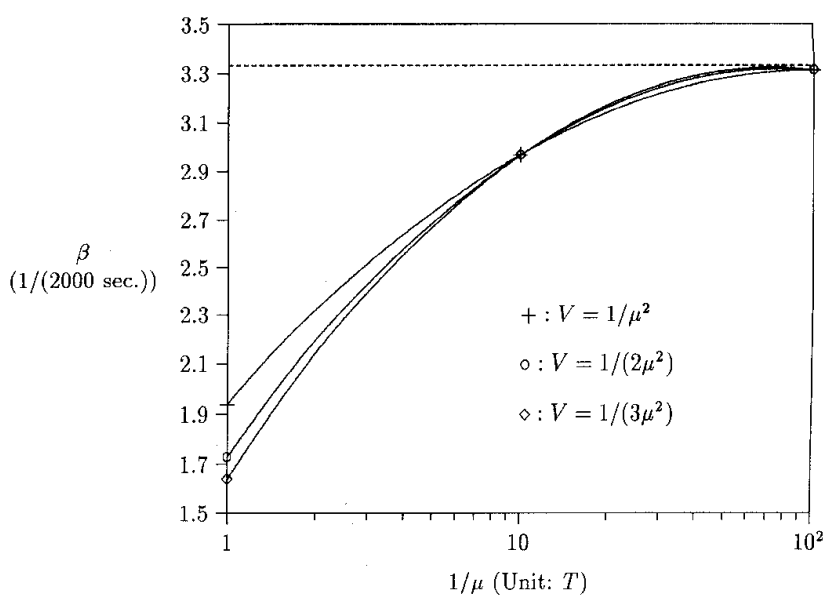

Fig. 4. Effect of $\mu$ on $\beta[T=30 \mathrm{~s}, \lambda=1 /(2000 \mathrm{~s})]$.

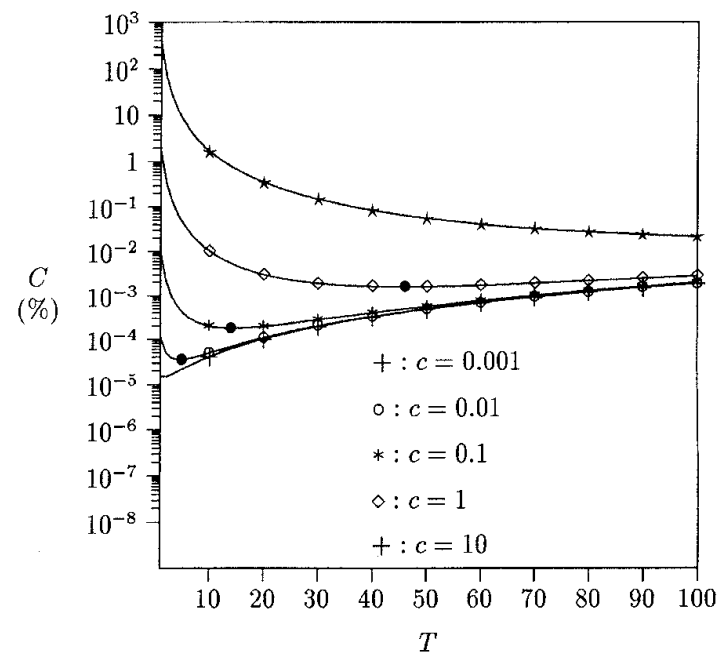

Fig. 5. Effect of $T$ on $C[1 / \mu \gg T, \lambda=1 /(2000 \mathrm{~s})]$.

specification. An analytic model is proposed to analyze the performance of the polling protocol. A cost function is derived, which can be used to estimate the optimal polling frequency. In the cost function, a cost factor $c$ is used to determine the relative costs for losing a call and the power consumption for transmission of polling messages. The value $c$ depends on the design of specific wireless local communication networks. For $c=0.001$, the polling costs are very low and $T$ should be small, perhaps every few seconds for a residential cordless system and every 30 seconds for a large wireless Centrex or PBX system. For $c=10$, the polling costs are high and $T$ should be greater than $100 \mathrm{~s}$.

\section{APPENDIX A}

Deriving THE Probability $\alpha$

Consider Fig. 1. Let $f_{m}$ be the density function of a user in an RA. The relationship between $t_{m}$ and $t$ is

$$
t_{m}=(i+1) T-t
$$


for some $i \geq 0$. Thus the density function $f$ for $t$ is

$$
f(t)=\sum_{i=0}^{\infty} f_{m}((i+1) T-t)
$$

Let $\lambda$ be the call arrival rate. Since the the call arrivals form a Poisson process and because of the excess life property [14] of the exponential distribution, the probability that $L$ calls arrive during the time period $X=t+\Delta$ is

$$
\operatorname{Pr}[L=l \mid X=x]=\frac{(\lambda x)^{l}}{l !} e^{-\lambda x} .
$$

Let $\alpha$ be the probability that there are call arrivals during the transition period $X$ of deregistration. The probability $\alpha^{*}=1-\alpha$ that no call arrive during $X$ is

$$
\begin{aligned}
\alpha^{*} & =\int_{t=0}^{T} \operatorname{Pr}[L=0 \mid X=t+\Delta] f(t) d t \\
& =e^{-\lambda \Delta} \int_{t=0}^{T}\left[\sum_{i=0}^{\infty} f_{m}((i+1) T-t)\right] e^{-\lambda t} d t .
\end{aligned}
$$

Let $\tau=(i+1) T-t$, then (10) is rewritten as

$$
\begin{aligned}
\alpha^{*}= & e^{-\lambda \Delta} \sum_{i=0}^{\infty} \int_{\tau=(i+1) T}^{i T} f_{m}(\tau) \\
& \times e^{-\lambda((i+1) T-\tau)} d((i+1) T-\tau) \\
= & e^{-\lambda \Delta} \sum_{i=0}^{\infty} e^{-(i+1) \lambda T} \int_{\tau=i T}^{(i+1) T} f_{m}(\tau) e^{\lambda \tau} d \tau
\end{aligned}
$$

If $f_{m}(t)$ is a Gamma density function $f_{\gamma, \eta}(t)$ with the shape parameter $\gamma$ and the scale parameter $\eta[7]$, then $f_{m}(t)=$ $f_{\gamma, \eta}(t)$ [see (2)] and the distribution function is $F_{m}(t)=$ $F_{\gamma, \eta}(t)=\int_{\tau=0}^{t} f_{\gamma, \eta}(\tau) d \tau$. Equation (11) is rewritten as

$$
\begin{aligned}
\alpha^{*}= & e^{-\lambda \Delta} \sum_{i=0}^{\infty} e^{-(i+1) \lambda T} \int_{\tau=i T}^{(i+1) T} \frac{\eta^{\gamma} \tau^{\gamma-1} e^{-\eta \tau}}{\Gamma(\gamma)}-e^{\lambda \tau} d \tau \\
= & e^{-\lambda \Delta} \sum_{i=0}^{\infty} e^{-(i+1) \lambda T}\left(\frac{\eta}{\eta-\lambda}\right)^{\gamma} \\
& \times \int_{\tau=i T}^{(i+1) T} \frac{(\eta-\lambda)^{\gamma} \tau^{-\gamma-1}}{\Gamma(\gamma)} e^{-(\eta-\lambda) \tau} d \tau \\
= & e^{-\lambda \Delta} \sum_{i=0}^{\infty} e^{-(i+1) \lambda T}\left(\frac{\eta}{\eta-\lambda}\right)^{\gamma} \\
& \times\left[F_{\gamma, \eta-\lambda}((i+1) T)-F_{\gamma, \eta-\lambda}(i T)\right]
\end{aligned}
$$

The values of $F_{\gamma, \eta-\lambda}(i T)$ and $F_{\gamma, \eta-\lambda}((i+1) T)$ can be obtained from the Gamma distribution table [7]. If $\gamma$ is a nonnegative integer, then from [16]

$$
F_{\gamma, \eta-\lambda}(t)=1-\sum_{j=0}^{\gamma-1} \frac{[(\eta-\lambda) t]^{j}}{j !} e^{-(\eta-\lambda) t}
$$

Equation (12) is rewritten as

$$
\alpha^{*}=e^{-\lambda \Delta}\left(\frac{\eta}{\eta-\lambda}\right)^{\gamma} \sum_{i=0}^{\infty} e^{-(i+1) \lambda T}
$$

$$
\begin{aligned}
& \times\left[\sum_{j=0}^{\gamma-1} \frac{[(\eta-\lambda) i T]^{j}}{j !} e^{-(\eta-\lambda) i T}\right. \\
& \left.-\sum_{j=0}^{\gamma-1} \frac{[(\eta-\lambda)(i+1) T]^{j}}{j !} e^{-(\eta-\lambda)(i+1) T}\right] \\
& =e^{-\lambda \Delta}\left(\frac{\eta}{\eta-\lambda}\right)^{\gamma} \\
& \times\left\{\sum_{j=0}^{\gamma-1} \frac{[(\eta-\lambda) T]^{j}}{j !}\right. \\
& \times\left\{\sum _ { i = 0 } ^ { \infty } e ^ { - ( i + 1 ) \lambda T } \left[i^{j} e^{-(\eta-\lambda) i T}\right.\right. \\
& \left.\left.\left.-(i+1)^{j} e^{-(\eta-\lambda)(i+1) T}\right]\right\}\right\} \\
& =e^{-\lambda \Delta}\left(\frac{\eta}{\eta-\lambda}\right)^{\gamma} \\
& \times\left\{\sum _ { j = 0 } ^ { \gamma - 1 } \frac { [ ( \eta - \lambda ) T ] ^ { j } } { j ! } \left[\sum_{i=0}^{\infty} i^{j} e^{-i \eta T} e^{-\lambda T}\right.\right. \\
& \left.\left.-\sum_{i=0}^{\infty}(i+1)^{j} e^{-(i+1) \eta T}\right]\right\} \\
& =e^{-\lambda \Delta}\left(\frac{\eta}{\eta-\lambda}\right)^{\gamma} \\
& \times\left\{\frac{e^{-\lambda T}-e^{-\eta T}}{1-e^{-\eta T}}+\sum_{j=1}^{\gamma-1} \frac{[(\eta-\lambda) T]^{j}}{j !}\right. \\
& \left.\times\left[\sum_{i=0}^{\infty} i^{j} e^{-i \eta T} e^{-\lambda T}-\sum_{i=0}^{\infty}(i+1)^{j} e^{-(i+1) \eta T}\right]\right\} \\
& =e^{-\lambda \Delta}\left(\frac{\eta}{\eta-\lambda}\right)^{\gamma} \\
& \times\left\{\frac{e^{-\lambda T}-e^{-\eta T}}{1-e^{-\eta T}}+\left(e^{-\lambda T}-1\right)\right. \\
& \left.\times\left[\sum_{j=1}^{\gamma-1} \frac{[(\eta-\lambda) T]^{j}}{j !}\left(\sum_{i=1}^{\infty} i^{j} e^{-i \eta T}\right)\right]\right\} .
\end{aligned}
$$

Note that

$$
\sum_{i=1}^{\infty} i^{j} e^{-i \eta T}=\left(\frac{e^{-\eta T}}{1-e^{-\eta T}}\right) \sum_{i=1}^{\infty} i^{j}\left(1-e^{-\eta T}\right)\left(e^{-\eta T}\right)^{i-1}
$$

where the summation term in the right-hand side is the $j$ th moment of the Geometric distribution with the probability function

$$
p_{k}=\left(1-e^{-\eta T}\right)\left(e^{-\eta T}\right)^{i-1} \quad \text { for } \quad i \geq 1
$$

and the moment generating function

$$
\Phi(s)=\frac{1-e^{-\eta T}}{e^{-s}-e^{-\eta T}} .
$$

The $j$ th moment of the geometric distribution can be obtained by differentiating (14) $j$ times with $s=0$. Thus (13) is 
rewritten as

$$
\begin{aligned}
\alpha=1 & -e^{-\lambda \Delta}\left(\frac{\eta}{\eta-\lambda}\right)^{\gamma} \\
& \times\left\{\frac{e^{-\lambda T}-e^{-\eta T}}{1-e^{-\eta T}}+\left(e^{-\lambda T}-1\right)\right. \\
& \left.\times\left\{\sum_{j=0}^{\gamma-1}\left\{\frac{e^{-\eta T}[(\eta-\lambda) T]^{j}}{j !\left(1-e^{-\eta T}\right)}\left[\left.\frac{d^{j} \Phi(s)}{d s^{j}}\right|_{s=0}\right]\right\}\right\}\right\} \\
=1- & e^{-\lambda \Delta}\left(\frac{\eta}{\eta-\lambda}\right)^{\gamma} \\
\times & \left\{\frac{e^{-\lambda T}-e^{-\eta T}}{1-e^{-\eta T}+\left(e^{-\lambda T}-1\right)}\right. \\
& \times \sum_{j=0}^{\gamma-1}\left\{\frac{[(\eta-\lambda) T]^{j}}{j !}\left[\left.\frac{d^{j}}{d s^{j}}\left(\frac{e^{-\eta T}}{e^{-s}-e^{-\eta T}}\right)\right|_{s=0}\right]\right\} .
\end{aligned}
$$

\section{APPENDIX B}

\section{DERIVING $\alpha$ WHEN $\mu \rightarrow 0$}

Consider the case when the user residual time in an RA is very long; i.e., $1 / \mu \rightarrow \infty$. From (15), we have

$$
\lim _{\mu \rightarrow 0} \alpha=\lim _{\eta \rightarrow 0} \alpha=1-e^{-\lambda \Delta}\left[\alpha_{1}+\left(e^{-\lambda T}-1\right) \alpha_{2}\right]
$$

where

$$
\begin{aligned}
\alpha_{1} & =\lim _{\eta \rightarrow 0}\left(\frac{\eta}{\eta-\lambda}\right)^{\gamma}\left(\frac{e^{-\lambda T}-e^{-\eta T}}{1-e^{-\eta T}}\right) \\
& =\lim _{\eta \rightarrow 0}\left(\frac{\eta}{\eta-\lambda}\right)^{\gamma}\left[\frac{e^{-\lambda T}-e^{-\eta T}}{-\sum_{k=0}^{\infty}(-\eta T)^{k}}\right] \\
& =\lim _{\eta \rightarrow 0}\left(\frac{1}{\eta-\lambda}\right)^{\gamma}\left[\frac{e^{-\lambda T}-e^{-\eta T}}{-\left(\frac{1}{\eta^{\gamma}}\right) \sum_{k=0}^{\infty}(-\eta T)^{k}}\right] \\
& =\left(-\frac{1}{\lambda}\right)^{\gamma}\left[\frac{1-e^{-\lambda T}}{\lim _{\eta \rightarrow 0}\left(\frac{T}{\eta^{\gamma-1}}\right)}\right] \\
& =0
\end{aligned}
$$

and

$$
\begin{aligned}
\alpha_{2}= & \lim _{\eta \rightarrow 0}\left(\frac{\eta}{\eta-\lambda}\right)^{\gamma} \\
& \times\left\{\sum_{j=0}^{\gamma-1} \frac{[(\eta-\lambda) T]^{j}}{j !}\left[\left.\frac{d^{j}}{d s^{j}}\left(\frac{e^{-\eta t}}{e^{-s}-e^{-\eta T}}\right)\right|_{s=0}\right]\right\} \\
= & \lim _{\eta \rightarrow 0}\left(\frac{\eta}{\eta-\lambda}\right)^{\gamma} \\
& \times\left\{\sum_{j=0}^{\gamma-1}[(\eta-\lambda) T]^{j}\left[\sum_{l=0}^{j} \frac{X_{j, l} e^{-\eta T}}{\left(1-e^{-\eta T}\right)^{l+1}}\right]\right\}
\end{aligned}
$$

where $X_{j, l}$ is a constant with respect to $\eta(0 \leq l \leq j<\gamma)$ and $X_{\gamma-1, \gamma-1}=1$. Equation (18) is rewritten as

$$
\alpha_{2}=\lim _{\eta \rightarrow 0}\left(\frac{1}{\eta-\lambda}\right)^{\gamma}
$$

$$
\begin{aligned}
& \times\left\{\sum_{j=0}^{\gamma-1}\left[\frac{T^{j} e^{-\eta T}}{(\eta-\lambda)^{\gamma-j}}\right]\right. \\
& \left.\times\left\{\sum_{l=0}^{j} \frac{X_{j, l}}{\left(\frac{1}{\eta^{\gamma}}\right)\left[-\sum_{k=1}^{\infty}(-\eta T)^{k}\right]^{l+1}}\right\}\right\} .
\end{aligned}
$$

Note that for $l, j<\gamma-1$,

$$
\lim _{\eta \rightarrow 0} \frac{X_{j, l}}{\left(\frac{1}{\eta^{\gamma}}\right)\left[-\sum_{k=1}^{\infty}(-\eta T)^{k}\right]^{l+1}}=0
$$

and (19) is re-uwritten as

$$
\begin{aligned}
\alpha_{2}= & \lim _{\eta \rightarrow 0}\left\{\left[\frac{T^{j} e^{-\eta T}}{(\eta-\lambda)^{\gamma-j}}\right]\right. \\
& \left.\quad \times\left\{\frac{X_{j, j}}{\left(\frac{1}{\eta^{\gamma}}\right)\left[-\sum_{k=1}^{\infty}(-\eta T)^{k}\right]^{j+1}}\right\}\right\}\left.\right|_{j=\gamma-1} \\
= & \left(\frac{T^{\gamma-1}}{-\lambda}\right)\left[\frac{1}{\left(\frac{1}{\eta^{\gamma}}\right)(\eta T)^{\gamma}}\right] \\
= & \frac{1}{-\lambda T}
\end{aligned}
$$

From (16), (17), and (20), we have

$$
\lim _{\mu \rightarrow 0} \alpha=1-\frac{e^{-\lambda \Delta}\left(1-e^{-\lambda T}\right)}{\lambda T} .
$$

\section{APPENDIX C}

Deriving The Probability $\beta$

Suppose that a user resides in an RA for a period $t_{m}$ and $E\left[t_{m}\right]=1 / \mu$. The number of re-registration messages sent from the HS to the BS is $\left|\frac{t_{m}}{T}\right|$. Let $\beta$ be the expected number of re-registration messages sent during a time unit. Then

$$
\begin{aligned}
\beta & =E\left[\left\lfloor\frac{t_{m}}{T}\right\rfloor\right] / E\left[t_{m}\right] \\
& =\mu \int_{t_{m}=0}^{\infty}\left\lfloor\frac{t_{m}}{T}\right\rfloor f_{m}\left(t_{m}\right) d t_{m} \\
& =\mu \sum_{i=0}^{\infty} \int_{\tau=i T}^{(i+1) T} f_{m}(\tau) d \tau \\
& =\mu \sum_{i=0}^{\infty} i\left[F_{m}((i+1) T)-F_{m}(i T)\right] \\
& =\mu \lim _{i \rightarrow \infty}\left[i F_{m}((i+1) T)-\sum_{j=1}^{i} F_{m}(j T)\right] \\
& =\mu \sum_{i=0}^{\infty}\left[1-F_{m}(i T)\right] .
\end{aligned}
$$

If $F_{m}$ is a Gamma distribution $F_{\gamma, \eta}$ with a nonnegative shape parameter $\gamma$ (where $\eta=\gamma \mu$ ), then (22) is rewritten as

$$
\begin{aligned}
\beta & =\mu \sum_{j=0}^{\gamma-1} \frac{(\gamma \mu T)^{j}}{j !}\left[\sum_{i=1}^{\infty} i^{j} e^{-i \gamma \eta T}\right] \\
& =\mu \sum_{j=0}^{\gamma-1}\left\{\frac{(\gamma \mu T)^{j}}{j !}\left[\left.\frac{d^{j}}{d s^{j}}\left(\frac{e^{-\gamma \mu T}}{e^{-s}-e^{-\gamma \mu T}}\right)\right|_{s=0}\right]\right\} .
\end{aligned}
$$


Since

$$
\frac{E\left[\frac{t_{m}}{T}\right]-T}{E\left[t_{m}\right]} \leq \beta \leq \frac{E\left[\frac{t_{m}}{T}\right]}{E\left[t_{m}\right]}
$$

and

$$
\lim _{t_{m} \rightarrow \infty} \frac{E\left[\frac{t_{m}}{T}\right]-T}{E\left[t_{m}\right]}=\frac{1}{T}-\lim _{t_{m} \rightarrow \infty} \frac{T}{E\left[t_{m}\right]}=\frac{1}{T}
$$

and

$$
\frac{E\left[\frac{t_{m}}{T}\right]}{E\left[t_{m}\right]}=\frac{1}{T}
$$

we have

$$
\lim _{\mu \rightarrow 0} \beta=\lim _{t_{m} \rightarrow \infty} \beta=\frac{1}{T}
$$

\section{REFERENCES}

[1] ANSI, "Personal access communications system air interface standard," Tech. rep. T1P1/95-035 and ANSI PN 3418, ANSI, 1995

[2] ANSI, "Personal access communications system unlicensed version B air interface standard," Tech. rep. T1P1/95-066 and ANSI PN, ANSI, 1995.

[3] Bellcore, "Generic criteria for version 0.1 wireless access communications systems (WACS) and supplement," Tech. rep. TR-INS-001313, Issue 1, Bellcore, 1994.

[4] Bellcore, "Advanced intelligent network 0.2 switching systems generic requirement," Tech. rep. GR-1298-CORE, Bellcore, 1995.

[5] EIA/TIA, "Cellular radio-telecommunications intersystem operations: Automatic roaming," Tech. report IS-41.3-B \}, EIA/TIA, 1991

[6] ETSI/TC, "Restoration procedures, version 4.2.0," Tech. rep. Recommendation GSM 03.07, ETSI, 1993.

[7] N. L. Johnson and S. Kotz, Continuous Univariate Distributions-1. New York: Wiley, 1970.

[8] J. Knapp, "Making user PCS a reality-A journey on the information highway," in 1st Annu. WINForum User PCS Workshop, 1994.

[9] Y.-B. Lin and A. Noerpel, "Implicit deregistration in a PCS network," IEEE Trans. Veh. Technol, vol. 43, no. 4, pp. 1006-1010, 1994.

[10] M. Mouly and M.-B. Pautet, The GSM System for Mobile Communications, Palaiseau, France, 1992.

[11] A. R. Noerpel, L. F. Chang, and Y.-B. Lin, "PACS contention algorithm for initial access and handoff," in 5th WINLAB Workshop, 1995, pp. $217-226$.

[12] A. R. Noerpel, L. F. Chang, and R. A. Ziegler, "PACS-UB, A protocol for the unlicensed spectrum," in IEEE ICC'95, 1995.
[13] A. R. Noerpel. R. Laborde, and K. Felix, "PACS-UB for use in unlicensed spectrum," in Ist Annu. WINForum User PCS Workshop, 1994

[14] S. M. Ross. Stochastic Processes. New York: Wiley, 1983.

[15] N. R. Sollenberger and H. W. Arnold, "Interoperable licensed and unlicensed wireless access for PCS," in IEEE 3rd Annu. Int. Conf. Universal Personal Communications Conf. Rec., 1994.

[16] L. Takacs, Introduction to the Theory of Queues. New York: Greenwood Press, 1961.

[17] R. A. Ziegler, A. R. Noerpel, L. F. Chang, and N. R. Sollenberger, "Low complexity hardware implementation for interoperable licensed \& unlicensed personal communications services: The PACS-UB system," in WINLAB Workshop, 1995.

Anthony R. Noerpel (S'76-M'84-SM'86), for a photograph and biography, see p. 727 of this issue.

Li Fung Chang (S'84-M'85-SM'90), for a photograph and biography, see p. 726 of this issue.

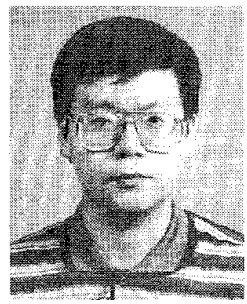

Yi-Bing Lin received the B.S.E.E. degree from the National Cheng Kung University, in 1983, and the Ph.D. degree in computer science from the University of Washington, Seattle, in 1990.

From 1990 to 1995 , he was with the Applied Research Area at Bell Communications Research (Bellcore), Morristown, NJ. In 1995, he was appointed a Full Professor with the Department and Institute of Computer Science and Information En gineering, National Chiao Tung University. His current research interests include design and analysis of personal communications services network, distributed simulation, and performance modeling. He is a Subject Area Editor of the Journal of Parallel and Distributed Computing, an Associate Editor of the International Journal in Computer Simulation, an Associate Editor of SIMULATION magazine, a member of the Editorial Board of the International Journal of Communications, a member of the Editorial Board of Computer Simulation Modeling and Analysts, Program Chair for the 8th Workshop on Distributed and Parallel Simulation, and General Chair for the 9th Workshop on Distributed and Parallel Simulation. 